\title{
Measurement of the $\mu^{+} \mu^{-}$Invariant Mass Spectra in $\mathbf{p}+\mathbf{p}$ and $\mathbf{p}+\mathrm{Au}$ Collisions at $\sqrt{s_{N N}}=200 \mathrm{GeV}$ with the Muon Telescope Detector at STAR
}

\author{
J. D. Brandenburg* for the STAR Collaboration \\ Rice University \\ Shandong University \\ Brookhaven National Laboratory \\ E-mail: jbrandenburg@bnl.gov
}

\begin{abstract}
Dileptons $\left(l^{+} l^{-}\right)$are produced during all stages of heavy-ion collisions (HIC) via various production mechanisms. Since leptons interact through the electromagnetic force, not via the strong force, they carry pristine information about the produced medium. For this reason, dileptons provide an essential tool for studying the properties of the strongly interacting medium produced in HICs. The installation of the Muon Telescope Detector (MTD) allows a measurement of the dimuon $\left(\mu^{+} \mu^{-}\right)$production over a large invariant mass range $\left(0<M_{\mu^{+} \mu^{-}}<4 \mathrm{GeV} / \mathrm{c}^{2}\right)$ for the first time at STAR. Data have been collected with the fully installed MTD from $p+p$ and $p+A u$ collisions at $\sqrt{s_{N N}}=200 \mathrm{GeV}$. These two data sets allow for new opportunities to measure the dimuon invariant mass spectra at STAR. In this talk, we will present new measurements of the $\mu^{+} \mu^{-}$invariant mass spectra in $\mathrm{p}+\mathrm{p}$ and $\mathrm{p}+\mathrm{Au}$ collisions at $\sqrt{s_{N N}}=200 \mathrm{GeV}$. The muon identification techniques employing deep neural networks and the novel background estimation methodology will be discussed. Finally, the invariant mass spectra will be compared with the hadronic cocktail and the physics will be discussed.
\end{abstract}

International Conference on Hard and Electromagnetic Probes of High-Energy Nuclear Collisions 30 September - 5 October 2018

Aix-Les-Bains, Savoie, France

\footnotetext{
${ }^{*}$ Speaker.
} 


\section{Introduction}

Dileptons $\left(l^{+} l^{-}\right)$provide an ideal probe of the hot and dense medium of strongly interacting matter produced in high energy heavy-ion collisions like those produced by the Large Hadron Collider (LHC) and the Relativistic Heavy-Ion Collider (RHIC) [1-4]. Since leptons are inert to the strong force, they carry pristine information about the whole evolution of the created system. Different production mechanisms related to different stages of the system can be distinguished through the invariant mass of produced pairs. The NA60 collaboration demonstrated that highprecision dimuon $\left(\mu^{+} \mu^{-}\right)$measurements are a valuable tool for studying the properties of the hot and dense medium [5-7]. The NA60 results motivate further study of the $\mu^{+} \mu^{-}$invariant mass spectra at RHIC energies. Since muons are difficult to differentiate from charged pions, a measurement of the $\mu^{+} \mu^{-}$invariant mass spectra over a wide range was not possible at STAR until recently, when the Muon Telescope Detector (MTD) was installed [8].

The MTD was installed to allow for identification of muons over a large momentum range for the first time at STAR. The plethora of hadrons produced in high-energy hadronic interactions makes hadron punch-through a significant source of background for muon identification with the MTD [9]. Identifying primordial $\mu^{+} \mu^{-}$pairs is further obscured by secondary muons resulting from weak decays of charged pions and kaons.

\section{The Muon Telescope Detector}

The Muon Telescope Detector is a detector built with multi-gap resistive plate chambers (MRPC) installed outside the STAR magnet return yoke steel at a radius of $\sim 410 \mathrm{~cm} \mathrm{[10].} \mathrm{The}$ magnet steel acts as the hadron absorber providing $\sim 5$ interaction lengths at its thickest. The MTD is segmented into 30 backlegs in the $\phi$ direction and 3 to 5 modules in the $z$ (beam line) direction. The MTD provides $\sim 45 \%$ coverage in $|\eta|<0.5$. The precise timing of the MTD, with a resolution of $\sigma \approx 100 \mathrm{ps}$, enables some of the slower hadron-punch through to be rejected. In addition to timing information, the MRPCs also provide $\sim 2 \mathrm{~cm}$ hit position resolution in the local $\mathrm{Y}$ and local $\mathrm{Z}$ directions. Altogether, the MTD and STAR Time Projection Chamber [11] provide 10 quantities that are used in the training of neural networks.

\section{Analysis Details}

Since the MTD has only a single layer of steel providing $\sim 5$ interaction lengths at its thickest, hadron contamination from punch-through pions, kaons, and protons is a major source of background in this analysis. Hadron punch-through is not the only significant source of contamination though. For the purpose of measuring primordial $\mu^{+} \mu^{-}$pairs, a significant background arises from secondary muons that are produced as singles - i.e. not in pairs. Significant backgrounds are also present from correlated secondary decay muons resulting from the decays of $\rho \rightarrow \pi^{+} \pi^{-} \rightarrow \mu^{+} \mu^{-}+X, K_{S}^{0} \rightarrow \pi^{+} \pi^{-} \rightarrow \mu^{+} \mu^{-}+X$, and $\phi \rightarrow K^{+} K^{-} \rightarrow \mu^{+} \mu^{-}+X$.

In order to obtain the most optimal muon identification, a neural network classifier was employed. In this study, the Toolkit for Multivariate Analysis (TMVA) was used to train the neural networks [12]. A grid-search strategy was used to determine the optimal deep multi-layer perceptron neural network architecture. Neural network architectures were scored based on their signal 


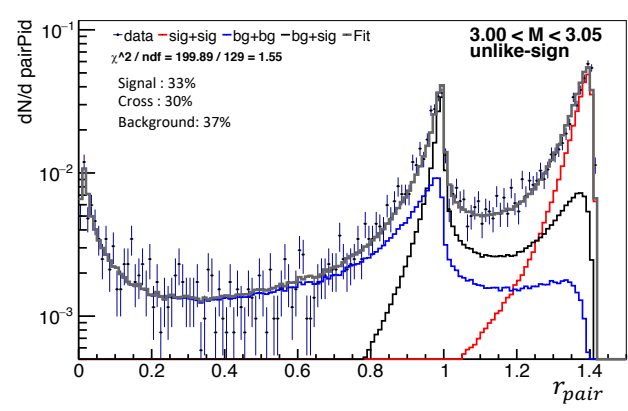

Figure 1: (color online) The results of the template-based fitting procedure of the $r_{\text {pair }}$ distribution for unlike-sign pairs in the invariant mass range $3.0<M_{\mu \mu}<3.05 \mathrm{GeV} / c^{2}$.

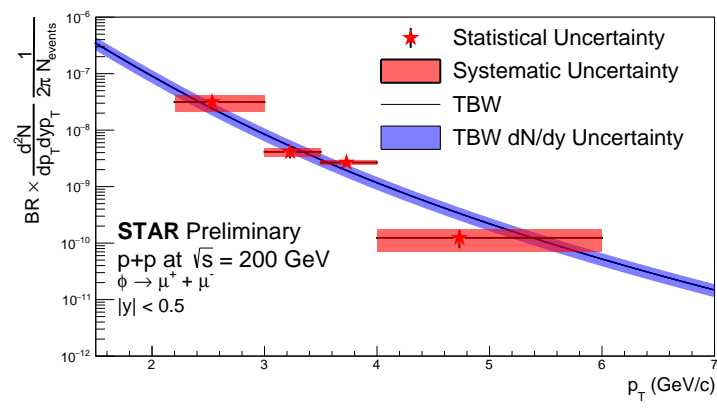

Figure 2: (color online) The branching ratio time the invariant yield of the $\phi \rightarrow \mu^{+}+\mu^{-}$measured in $\mathrm{p}+\mathrm{p}$ collisions at $\sqrt{s}=200 \mathrm{GeV}$. The statistical uncertainties are shown in vertical bars and the systematic uncertainties are shown in shaded boxes.

versus background rejection power, their complexity (number of neurons) and the shape of the discriminator output. The highest scoring network architectures had at least two hidden layers containing $\mathrm{N}+10$ and $\mathrm{N}+12$ neurons each ( $\mathrm{N}=10$ is the number of input variables).

The output of the muon identification neural network is a single discriminator value which gives values close to 1.0 for muons and values close to 0.0 for background tracks. Muon pairs are identified using $r_{\text {pair }}$ which is combination of the neural network response of the two daughter tracks, defined as $r_{\text {pair }}=\sqrt{r_{a}^{2}+r_{b}^{2}}$, where $r_{a}$ and $r_{b}$ are the neural network responses of the two tracks, $a$ and $b$, that make up the pair. This combination of the two daughter track neural network responses provides a single discriminator for identifying muon pairs from background pairs. Given two possible track categories (signal and background) there are three possible pair combinations: signal pairs $=$ signal + signal tracks, cross pairs $=$ signal + background tracks, and background pairs $=$ background + background tracks. The construction of the $r_{\text {pair }}$ variable ensures that $\mu^{+} \mu^{-}$ pairs, i.e. signal pairs, give $r_{\text {pair }} \approx \sqrt{2}$. Background pairs on the other-hand, yield an $r_{\text {pair }} \approx 0$ making the majority of background pairs relatively easy to reject. However, cross pairs will yield an $r_{\text {pair }} \approx 1.0$ with a non-trivial contribution from the tail polluting the signal region.

The amount of non-signal contribution from background and cross pairs is estimated using a template-based fit to the $r_{\text {pair }}$ distribution. Templates of the neural network response for signal and background tracks are parameterized as a function of the track $p_{T}, \eta$, and $\phi$. The single track neural network templates are then combined into templates of the $r_{\text {pair }}$ response via:

$$
\begin{aligned}
T_{\text {background }}^{\text {pair }} & \left(p_{T, 1}, \eta_{1}, \phi_{1}, p_{T, 2}, \eta_{2}, \phi_{2}\right)= \\
& \left(T_{\text {background }}^{\text {single-track }}\left(p_{T, 1}, \eta_{1}, \phi_{1}, Y_{\pi}\left(p_{T, 1}\right), Y_{K}\left(p_{T, 1}\right), Y_{p}\left(p_{T, 1}\right)\right)\right. \\
& \left.\otimes T_{\text {background }}^{\text {single-track }}\left(p_{T, 2}, \eta_{2}, \phi_{2}, Y_{\pi}\left(p_{T, 2}\right), Y_{K}\left(p_{T, 2}\right), Y_{p}\left(p_{T, 2}\right)\right)\right) \cdot w\left(p_{T, 1}, p_{T, 2}\right)
\end{aligned}
$$




$$
\begin{gathered}
T_{\text {cross }}^{\text {pair }}\left(p_{T, 1}, \eta_{1}, \phi_{1}, p_{T, 2}, \eta_{2}, \phi_{2}\right)= \\
\left(T_{\text {background }}^{\text {single-track }}\left(p_{T, 1}, \eta_{1}, \phi_{1}, Y_{\pi}\left(p_{T, 1}\right), Y_{K}\left(p_{T, 1}\right), Y_{p}\left(p_{T, 1}\right)\right)\right. \\
\left.\otimes T_{\text {signal }}^{\text {single-track }}\left(p_{T, 2}, \eta_{2}, \phi_{2}\right)\right) \cdot w\left(p_{T, 1}, p_{T, 2}\right) \\
T_{\text {signal }}^{\text {pair }}\left(p_{T, 1}, \eta_{1}, \phi_{1}, p_{T, 2}, \eta_{2}, \phi_{2}\right)= \\
\left(T_{\text {signal }}^{\text {single-track }}\left(p_{T, 1}, \eta_{1}, \phi_{1}\right) \otimes T_{\text {signal }}^{\text {single-track }}\left(p_{T, 2}, \eta_{2}, \phi_{2}\right)\right) \cdot w\left(p_{T, 1}, p_{T, 2}\right)
\end{gathered}
$$

where $T_{\text {signal }}^{\text {single-track }}(\ldots)$ and $T_{\text {background }}^{\text {single-track }}(\ldots)$ are the single track template shapes for signal and background, respectively and $w$ is a weight for ensuring proper $p_{T}$ correlation between daughter tracks when building the templates. Each single-track template is a function of the daughter track's kinematic variables $\left(p_{T}, \eta, \phi\right)$. In order to take into account the variations in background template shape due to different hadron contamination levels, the single-track background template is also a function of the relative yields of $\pi, \mathrm{K}$, and $p\left(Y_{\pi}, Y_{K}\right.$, and $Y_{p}$ respectively) for the given $p_{T}$ bin. Figure 1 shows an example of the $r_{\text {pair }}$ distribution fit to:

$$
f\left(r_{\text {pair }}\right)=Y_{\text {background }} \times T_{\text {background }}^{\text {pair }}+Y_{\text {cross }} \times T_{\text {cross }}^{\text {pair }}+Y_{\text {signal }} \times T_{\text {signal }}^{\text {pair }}
$$

where $Y_{\text {background }}, Y_{\text {cross }}$, and $Y_{\text {signal }}$ are the free fit parameters corresponding to the yield of background, cross, and signal pairs, respectively. Fig. 1 shows an example of a fit to the $r_{\text {pair }}$ distribution in an invariant mass bin under the $J / \psi$ peak. The $r_{\text {pair }}$ distribution in data is reasonably well described by the fit. As can be seen in Fig. 1 each of the three contributions can be well separated in the $r_{\text {pair }}$ distribution, making it possible to unambiguously extract the signal yield. The remaining background from combinatorial $\mu^{+} \mu^{-}$pairs present in the unlike-sign distribution is estimated using the same procedure in the like-sign distribution to extract the final signal distribution.

\section{Results}

The neural network based muon identifiaction made the first STAR measurement of the $\phi$ meson spectra through the $\phi \rightarrow \mu^{+}+\mu^{-}$decay channel possible. The branching ratio times invariant yield (shown in Fig. 2) was measured in $\mathrm{p}+\mathrm{p}$ collisions at $\sqrt{s}=200 \mathrm{GeV}$ in four transverse momentum bins. The $\phi \rightarrow \mu^{+}+\mu^{-}$invariant yield times branching ratio was found to be in good agreement with previous measurements through the $\phi \rightarrow K^{+}+K^{-}$decay channel [13, 14].

Additionally, with these techniques the $\mu^{+} \mu^{-}$invariant mass spectra was measured in $\mathrm{p}+\mathrm{p}$ and minimum bias $\mathrm{p}+\mathrm{Au}$ collisions $\left(\left\langle N_{\text {coll }}\right\rangle^{0-100 \%}=4.7 \pm 0.3\right)$ at $\sqrt{s_{\mathrm{NN}}}=200 \mathrm{GeV}$ for the first time with STAR. The fully corrected $\mu^{+} \mu^{-}$invariant mass spectra are shown in Fig. 3 and Fig. 4 for the $p+p$ and $p+A u$ data, respectively. The ratio of data to hadronic cocktail is shown in Fig. 5 and Fig. 6 for $\mathrm{p}+\mathrm{p}$ and $\mathrm{p}+\mathrm{Au}$, respectively. There is good agreement across the entire invariant mass range within the substantial uncertainties. At masses just above threshold mass, the combination of poor efficiency and significant, focused hadron contamination from $K_{S}^{0} \rightarrow \pi^{+}+\pi^{-}$and $\phi \rightarrow K^{+}+K^{-}$ makes it difficult to resolve any dimuon signal. 


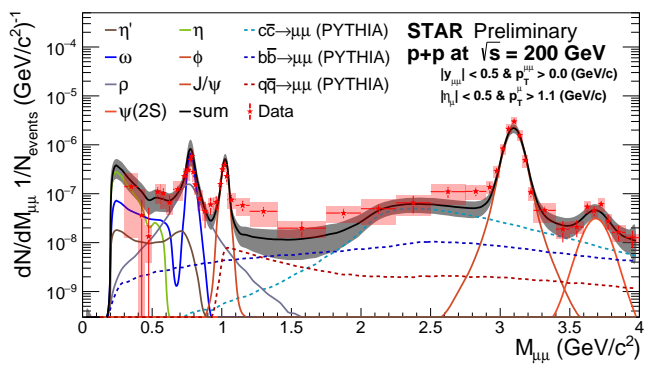

Figure 3: (color online) The $\mu^{+} \mu^{-}$invariant mass spectra in $\mathrm{p}+\mathrm{p}$ collisions at $\sqrt{s}=200 \mathrm{GeV}$. The statistical uncertainties are shown in vertical bars and the systematic uncertainties are shown in shaded boxes.

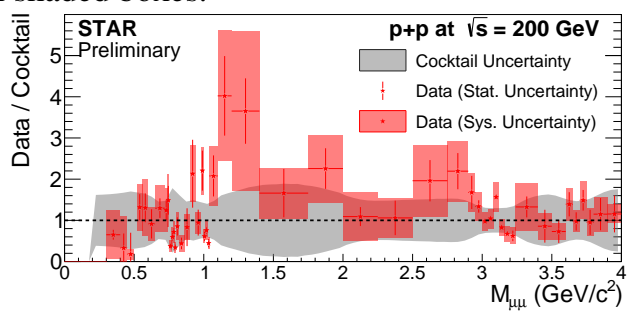

Figure 5: (color online) The ratio of the $\mu^{+} \mu^{-}$invariant mass spectra in $\mathrm{p}+\mathrm{p}$ collisions at $\sqrt{s}=200 \mathrm{GeV}$ over the total hadronic cocktail.

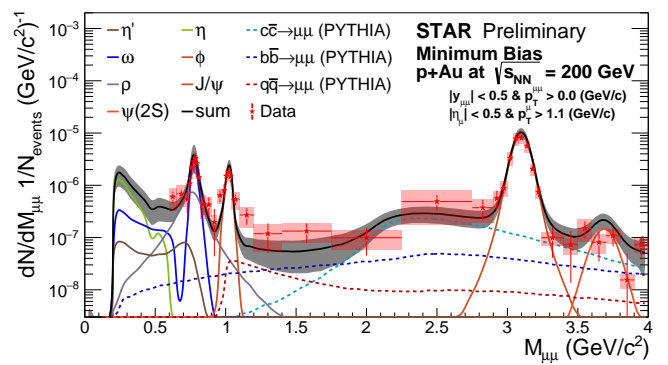

Figure 4: (color online) The $\mu^{+} \mu^{-}$invariant mass spectra in $\mathrm{p}+\mathrm{Au}$ collisions at $\sqrt{s_{\mathrm{NN}}}=200$ $\mathrm{GeV}$. The statistical uncertainties are shown in vertical bars and the systematic uncertainties are shown in shaded boxes.

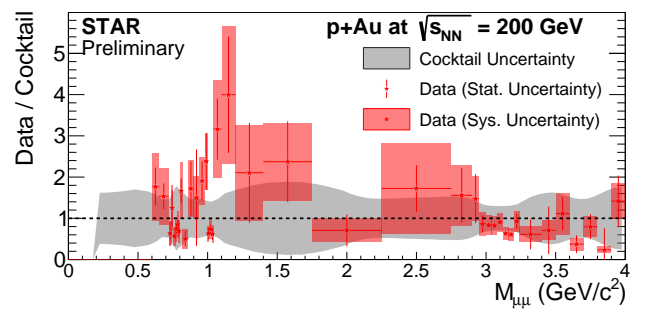

Figure 6: (color online) The ratio of the $\mu^{+} \mu^{-}$invariant mass spectra in $\mathrm{p}+\mathrm{Au}$ collisions at $\sqrt{s_{\mathrm{NN}}}=200 \mathrm{GeV}$ over the total hadronic cocktail.

\section{Summary}

In this contribution, we reported the first STAR measurements of the the $\mu^{+} \mu^{-}$invariant mass spectra in $\mathrm{p}+\mathrm{p}$ and $\mathrm{p}+\mathrm{Au}$ collisions at $\sqrt{s_{\mathrm{NN}}}=200 \mathrm{GeV}$. Significant backgrounds from hadron contamination motivated the use of deep neural networks for maximizing the muon identification capabilities of the MTD. The single-track muon identification information was combined for pair identification and a novel background estimation technique was developed to extract the $\mu^{+} \mu^{-}$ signal in the presence of significant correlated background sources. Using these techniques the $\mu^{+} \mu^{-}$invariant mass spectra were measured in $\mathrm{p}+\mathrm{p}$ and $\mathrm{p}+\mathrm{Au}$ collisions at $\sqrt{s_{\mathrm{NN}}}=200 \mathrm{GeV}$ for the first time with STAR. The spectra in $p+p$ and $p+A u$ collisions are found to be consistent with their respective simulated hadronic cocktails. The improved muon identification capabilities provided by the deep neural network also allowed the first STAR measurement of the $\phi$ through the $\mu^{+} \mu^{-}$decay channel. These measurements provide a baseline for future measurement in $\mathrm{Au}+\mathrm{Au}$ collisions at STAR.

Acknowledgments: This work was funded by the U.S. DOE Office of Science under contract No. DE-FG02-10ER41666.

\section{References}

[1] BRAHMS Collaboration, Quark Gluon Plasma an Color Glass Condensate at RHIC? The 
perspective from the BRAHMS experiment, Nuclear Physics A 757 (2005) 1.

[2] PHOBOS Collaboration, The PHOBOS Perspective on Discoveries at RHIC, Nuclear Physics A 757 (2005) 28.

[3] STAR Collaboration, Experimental and Theoretical Challenges in the Search for the Quark Gluon Plasma: The STAR Collaboration's Critical Assessment of the Evidence from RHIC Collisions, Nuclear Physics A 757 (2005) 102.

[4] PHENIX Collaboration, Formation of dense partonic matter in relativistic nucleus-nucleus collisions at RHIC: Experimental evaluation by the PHENIX collaboration, Nuclear Physics A 757 (2005) 184.

[5] NA60 Collaboration, R. Arnaldi, K. Banicz, K. Borer, J. Castor, B. Chaurand et al., NA60 results on thermal dimuons, The European Physical Journal C 61 (2009) 711.

[6] P. M. Hohler and R. Rapp, Realistic Implementation of Massive Yang Mills for rho and al Mesons, Physical Review D 89 (2014) .

[7] R. Rapp and H. van Hees, Thermal Dileptons as Fireball Thermometer and Chronometer, Physics Letters B 753 (2016) 586.

[8] L. Ruan, G. Lin, Z. Xu, K. Asselta, H. F. Chen, W. Christie et al., Perspectives of a Midrapidity Dimuon Program at RHIC: A Novel and Compact Muon Telescope Detector, Journal of Physics G: Nuclear and Particle Physics 36 (2009) 095001.

[9] T. C. Huang, R. Ma, B. Huang, X. Huang, L. Ruan, T. Todoroki et al., Muon Identification with Muon Telescope Detector at the STAR Experiment, Nuclear Instruments and Methods in Physics Research Section A: Accelerators, Spectrometers, Detectors and Associated Equipment 833 (2016) 88.

[10] C. Yang, X. J. Huang, C. M. Du, B. C. Huang, Z. Ahammed, A. Banerjee et al., Calibration and performance of the STAR Muon Telescope Detector using cosmic rays, Nuclear Instruments and Methods in Physics Research Section A: Accelerators, Spectrometers, Detectors and Associated Equipment 762 (2014) 1.

[11] M. Anderson, J. Berkovitz, W. Betts, R. Bossingham, F. Bieser, R. Brown et al., The STAR Time Projection Chamber: A Unique Tool for Studying High Multiplicity Events at RHIC, Nuclear Instruments and Methods in Physics Research Section A: Accelerators, Spectrometers, Detectors and Associated Equipment 499 (2003) 659.

[12] A. Hoecker, P. Speckmayer, J. Stelzer, J. Therhaag, E. von Toerne and H. Voss, TMVA: Toolkit for Multivariate Data Analysis, PoS ACAT (2007) 040 [phys i cs / 0703039 ].

[13] T. Zebo, Y. Li, R. Li-Juan, S. Ming, L. Cheng, C. Hong-Fang et al., The Statistical Origin of Constituent-Quark Scaling in QGP Hadronization, Chinese Phys. Lett. 30 (2013) 031201.

[14] STAR Collaboration, $\phi$ meson production in $A u+A u$ and $p+p$ collisions at $\sqrt{s_{N N}}=200$ GeV, Physics Letters B 612 (2005) 181. 